Published online 2017 April 13.

Abstract

\title{
Safety Considerations in Cardiovascular Magnetic Resonance Imaging
} Studies

\section{Maryam Moradi ${ }^{1, *}$}

${ }^{1}$ Isfahan University of Medical Sciences, Isfahan, Iran

"Corresponding author: Maryam Moradi, Isfahan University of Medical Sciences, Isfahan, Iran. E-mail: moradi@med.mui.ac.ir

Received 2016 December 21; Accepted 2017 February 08.

\begin{abstract}
The aim of this review is focusing on the most important safety issues in cardiovascular MRI (magnetic resonance imaging) which are categorized as: 1-safety items in patients who referred for stress cardiac MR studies. 2-Safety consideration that we must know when a patient with cardiovascular devices referred for any (cardiovascular or non-cardiovascular) MRI with focus on new protocols according to update or old devices. 3-Review safety issues in the use of MR contrast agent.
\end{abstract}

This is an abstract presented in the 33rd Iranian congress of radiology (ICR) and the 15th congress of Iranian radiographic science association (IRSA). 